\title{
Can we use short recordings for assessment of dynamic cerebral autoregulation? A sensitivity analysis study in acute ischaemic stroke and healthy subjects
}

\author{
Kannakorn Intharakham ${ }^{1}$, Ronney B Panerai ${ }^{1,2}$, Emmanuel Katsogridakis ${ }^{1}$, Man Y Lam ${ }^{1}$, Osian Llwyd ${ }^{1}$, \\ Angela S M Salinet ${ }^{1,3}$, Ricardo C. Nogueira ${ }^{3}$, Victoria Haunton ${ }^{1,2}$ and Thompson G Robinson ${ }^{1,2}$ \\ ${ }^{1}$ Department of Cardiovascular Sciences, Cerebral Haemodynamics in Ageing and Stroke Medicine (CHIASM) Research \\ Group, University of Leicester, Leicester, United Kingdom \\ ${ }^{2}$ National Institute for Health Research Leicester Biomedical Research Centre, University of Leicester, Leicester, United \\ Kingdom \\ ${ }^{3}$ Neurology Department, Hospital das Clinicas, School of Medicine, University of São Paulo, São Paulo, Brazil
}

Keywords: dynamic cerebral autoregulation, transfer function analysis, cerebral blood flow

\begin{abstract}
Objective: It is unclear whether the duration of recordings influences estimates of dynamic cerebral autoregulation (dCA). Therefore, we performed a retrospective study of the effects of reducing recording durations on dCA estimates; with the potential to inform recording duration for reliable estimates in challenging clinical populations.
\end{abstract}

Approach: Seventy-eight healthy control subjects and 79 acute ischaemic stroke (AIS) patients were included. Cerebral blood flow velocity was recorded with transcranial Doppler and continuous blood pressure with the Finapres device. The autoregulation index (ARI), derived with transfer function analysis, was calculated for recording durations at one-minute intervals between 1 and 5 minutes using the same starting point of each recording.

Main results: Though recording duration did not affect the overall ARI value, when compared to control subjects, AIS patients had significantly lower ARI values for durations between 3 and $5(p<0.0001)$, but not 1 and 2 minutes. The intraclass correlation coefficient of all participants, for reproducibility of the five recording durations, was 0.69. AIS patients classified as having impaired cerebral autoregulation $(\mathrm{CA} ; \mathrm{ARI} \leq 4)$ at $5 \mathrm{~min}$, had a $7.1 \%$ rate of false negatives for both 4 and 3 min recordings, reaching $42.9 \%$ for 1 min recording. The percentage of false-positives also increased with reduced recording durations (from $0 \%$ at 5 to $16.2 \%$ at 1 minute).

Significance: Reducing recording durations from 5 to $3 \mathrm{~min}$ can still provide reliable estimates of ARI, and may facilitate CA studies in potentially medically unstable AIS patients, as well as in other patient groups. 


\section{Introduction}

Cerebral autoregulation (CA) is a complex mechanism that tends to maintain constant cerebral blood flow $(\mathrm{CBF})$ despite changes in cerebral perfusion pressure (CPP) in the range of 60 to $150 \mathrm{mmHg}$ (Lassen, 1959). CA has been conceptualised as either a static or a dynamic process (Aaslid et al 1989; Tiecks et al 1995; Panerai 1998). Dynamic CA (dCA) represents the CBF transient response, over a few seconds, to sudden changes in blood pressure (BP) (Aaslid et al., 1989), while static CA (sCA) represents the steady state behaviour of the BP-CBF relationship over several minutes (Panerai, 1998; Tiecks et al., 1995).

One of the main advantages of $\mathrm{dCA}$, when compared to $\mathrm{sCA}$, is the ability to perform physiological and clinical assessments of cerebral haemodynamics parameters, using non-invasive measurements at rest, based on spontaneous fluctuations in $\mathrm{BP}$ and $\mathrm{CBF}$, usually estimated as $\mathrm{CBF}$ velocity (CBFV) with transcranial Doppler (TCD) ultrasound (Panerai, 2008). As a broader marker of cerebrovascular health, dCA has been shown to be affected by a range of different pathologies including stroke, traumatic brain injury, neonatal prematurity and intracranial hypertension (Aries et al., 2010; Czosnyka et al., 2008; Panerai et al., 2002; Panerai et al., 2016b).

In a recent White Paper (Claassen et al., 2016), the Cerebral Autoregulation Network (CARnet) has proposed the standardisation of TFA settings and parameters, aiming to achieve greater homogeneity and reliability in TFA applications to dCA assessment. One main recommendation was for recordings at rest to be of a minimum duration of 5 min (Claassen et al., 2016). Unfortunately, in clinical studies, good quality recordings of $5 \mathrm{~min}$ are not always possible due to patient movement, environmental noise or patient discomfort. Abiding to the CARNet's recommendations in these cases inevitably jeopardises individualised care, due to the need to reject recordings shorter than $5 \mathrm{~min}$. Although the literature reports a wide range of recording durations, from as long as $20 \mathrm{~min}$ (Tang et al., 2008; Mahdi et al., 2017; Chi et al., 2017), down to 1 min (Nakagawa et al., 2009; Carey et al., 2001; Carey et al., 2003; Panerai et al., 2001; Puppo et al., 2008), there is a lack of systematic studies describing the influence of recording duration on the TFA outcome parameters of interest. To address this limitation, we have retrospectively studied the influence of recording duration from both healthy subjects and acute ischaemic stroke (AIS) patients, including all subtypes were recorded. By reducing recording duration from the standard $5 \mathrm{~min}$, to durations of 4, 3, 2, and $1 \mathrm{~min}$, we tested the hypothesis that one of the main parameters derived from TFA, namely the ARI index, is not affected by shorter recordings, in either population. 


\section{Methods}

\subsection{Subjects and measurements}

Data used for this study were extracted from the Leicester Cerebral Haemodynamics Database (Patel et al., 2016), which comprises a large number of recordings performed in both healthy subjects and AIS patients (Llwyd et al., 2018). All studies that contributed to the database had local research Ethics Committee approval (Haunton, 2014; Katsogridakis et al., 2011; Lam et al., 2019; Llwyd et al., 2017; Nogueira et al., 2013; Salinet et al., 2014) and all subjects provided written informed consent. These studies had high homogeneity in protocols for inclusion and exclusion criteria, as well as measurement procedures (Brodie et al., 2009; Saeed et al., 2013; Atkins et al., 2010). In brief, healthy controls did not have a history or symptoms of any neurological, cardiovascular or respiratory disease. AIS patients, diagnosis confirmed by neuroimaging, were admitted to the University Hospitals of Leicester NHS Trust within $48 \mathrm{~h}$ of mild- (defined as National Institute of Health Stroke Scale [NIHSS] <8) -tomoderate stroke (NIHSS 8-15) (Wahlgren et al., 2007)). Exclusion criteria included any medical history or current evidence of myocardial infarction, renal failure, respiratory disease, or atrial fibrillation. All healthy participants were asked to avoid alcohol, caffeine, and nicotine for at least $4 \mathrm{~h}$ before attending the University of Leicester's Cerebral Haemodynamics in Ageing and Stroke Medicine (CHiASM) laboratory which is of controlled temperature $\left(20-24^{\circ} \mathrm{C}\right)$ and free from visual and auditory distraction. Bilateral insonation of the middle cerebral arteries (MCAs) was performed using TCD with 2MHz TCD probes (Viasys Companion III; Viasys Healthcare), which were secured in place using a head-frame in the position of maximum comfort for study participants. The MCAs were accurately identified, with maximal reflected signal power at depths of $45-55 \mathrm{~mm}$. Beat-to beat BP was continuously recorded using the Finometer ${ }^{\circledR}$ device (FMS, Finapres Measurement Systems, Arnhem, Netherlands), which was attached to the middle finger of the non-dominant hand in healthy subjects and the non-hemiparetic hand in AIS patients. The servo-correcting mechanism of the Finometer ${ }^{\circledR}$ was switched on for calibration and off prior to measurements. Heart rate (HR) was recorded using a 3-lead electrocardiogram (ECG) and end-tidal $\mathrm{CO}_{2}\left(\mathrm{EtCO}_{2}\right)$ was measured with nasal cannula (Salter Labs) via a capnograph (Capnocheck Plus). Brachial BP was measured with a sphygmomanometer (OMRON Model 705IT), and used to calibrate the Finometer ${ }^{\circledR}$ recordings. A baseline recording with subjects lying supine at rest was performed with a minimum of 5 min duration.

\subsection{Data analysis}

All signals were simultaneously recorded on the PHYSIDAS data acquisition system (Department of Medical Physics, University Hospitals of Leicester) at a sampling rate of 500Hz. BP was calibrated at the beginning of each recording. All recordings with at least 5min duration were visually inspected and narrow artefacts $(<100 \mathrm{~ms})$ were removed by linear interpolation. Smaller artefacts in the CBFV signals 
were removed with a median filter. All signals were then low-pass filtered with a zero-phase eight-order Butterworth filter with cut-off frequency of $20 \mathrm{~Hz}$. The R wave of the ECG was automatically detected. The beat-to-beat HR sequence was visually inspected and manually corrected in case of missed marks or presence of spikes. The mean values of $\mathrm{BP}, \mathrm{CBFV}, \mathrm{HR}$ and $\mathrm{EtCO}_{2}$ were calculated for each cardiac cycle. The end-tidal value of each breath was detected in the capnographic signal, linearly interpolated, and resampled with each cardiac cycle. Beat-to-beat parameters were spline interpolated and re-sampled at $5 \mathrm{~Hz}$ to generate signals with a uniform time-base.

Transfer function analysis of the linear relationship between BP and CBFV was estimated using Welch's method. For a five-minute recording, segments with duration of 102.4s (512 samples) were multiplied by a cosine window and the auto- and cross-spectrum were calculated with the FFT algorithm, using 50\% superposition as recommended by CARNet's White Paper (Claassen et al., 2016). After obtaining the amplitude and phase frequency responses, the inverse FFT was used to obtain the impulse response that was integrated to produce the CBFV step response to a sudden change in $\mathrm{BP}$ (Panerai, 2008). ARI was extracted by comparison of the CBFV step response to the template curves proposed by Tiecks et al. (1995); (Panerai et al., 1998). ARI estimates were not accepted if the average coherence function in the frequency interval $0.15-0.25 \mathrm{~Hz}$ was lower than the $95 \%$ confidence limit, or the normalised mean square error (NMSE) was below the critical limit of NMSE $=0.3$ for fitting the step response to Tieck's model (Panerai et al., 2016a). All healthy controls selected from the database had ARI $\geq 5$ taking into account that normal average ARI = 5 (Tiecks et al., 1995; Deegan et al., 2010; Subudhi et al., 2015). In patients, values of ARI $\leq 4$ were adopted as the criterion for impaired dCA (Patel et al., 2016; Caldas et al., 2017; Caldas et al., 2019).

Dynamic CA was modelled using transfer function analysis (TFA), adopting spontaneous fluctuations in BP as input and corresponding fluctuations in CBFV as output, has become the most common approach to assess dCA at rest, in both physiological and clinical studies (Claassen et al., 2016; Zhang et al., 1998; Giller, 1990). In the frequency domain, the main parameters derived with TFA are the coherence, gain (or amplitude) and phase frequency responses. The coherence function, varying between zero and 1, represents the fraction of output power that can be linearly explained by the input power (or variance) at each frequency (Bendat and Piersol, 2011). Estimates of gain and phase can be accepted as reliable markers of dCA only at frequencies where coherence is above its $95 \%$ confidence limit (Claassen et al., 2016). Non-significant values of coherence can result from a low signal-to-noiseratio, a non-linear relationship between $\mathrm{BP}$ and $\mathrm{CBFV}$, or when multiple variables are influencing CBFV. Moreover, using the inverse Fourier transform of the gain and phase, it is possible to estimate the CBFV step response to BP changes in the time domain (Panerai et al., 1998; Claassen et al., 2016). This function can be compared to the 10 template curves proposed by Tiecks et al (1995) to provide an estimate of the autoregulation index (ARI), which varies between zero (absence of CA) to 9 (best observed CA). A threshold value of ARI $\leq 4$ was adopted by previous studies to classify dCA as impaired, when using 5 min recording durations (Patel et al., 2016; Caldas et al., 2017). 


\subsection{Durations and segments analysis}

To assess the influence of shorter recording durations in the first five to one minutes of recordings on ARI estimates, the following combinations of number of segments and window durations were adopted as the reference condition: $4 \min$ (4x512 samples); 3 min (3x512 samples); 2 min (4x256 samples); and $1 \mathrm{~min}$ (4x 128 samples), with 50\% superposition in all cases. The influence of reducing window duration was assessed separately for the $3 \mathrm{~min}$ and 2 min using 256 samples and 128 samples respectively, as described below.

\subsection{Statistical analysis}

All data were tested for normality with the Shapiro-Wilk test. For simplicity, only the right hemisphere data was considered in healthy controls and only the affected hemisphere in AIS patients. For nonGaussian parameters, the Friedman test was used to assess the effect of shortening recording duration in each population. Reliability of successive reductions in recording duration was expressed by the intraclass correlation coefficient (ICC) calculated using the (1, 1) model (Shrout and Fleiss, 1979; Weir, 2005). Agreement between parameter values from recordings with different durations was assessed with the bias and limits of agreement and represented with Bland-Altman plots (Bland and Altman, 1986). Inter-group comparisons were assessed with the Mann-Whitney $U$ test. The interaction between the effects of disease and recording duration was assessed with a 2-way ANOVA. A value of $p<0.05$ was adopted to indicate statistical significance. Statistical analyses were performed with GraphPad Prism 7 and SPSS Statistics 25.

\section{Results}

Seventy-eight healthy controls and 79, mainly mild severity $(n=67)$ and moderate stroke $(n=12)$, AIS patients were initially included, with demographic and physiological characteristics given in Table 1. CBFVs were significantly higher in healthy controls compared to AIS patients at right and affected sides $(p=0.0002)$ and left and unaffected sides $(p<0.0001)$ respectively, whereas BP and HR were of lower values $(\mathrm{p}<0.0001)$. EtCO 2 was significantly lower in AIS patients $(\mathrm{p}<0.0001)$.

\subsection{Effect of shortening recording duration on population values}

Following application of the ARI acceptance criteria (see Methods), complete data for the five different recording durations were available for 67 control subjects and 75 AIS patients. Shorter recording duration did not have a significant effect on ARI for both AIS $(p=0.13)$ and control group $(p=0.068)$, though significant differences in ARI between populations were only maintained with recording durations down to $3 \mathrm{~min}$ (Figure. 1). For the combined study population, a 2-way ANOVA confirmed a strong effect of disease $(p<0.0001)$, but did not show an effect of recording duration on the ARI $(\mathrm{p}=0.56)$, or an interaction between these factors. 


\subsection{Intra-subject reproducibility with shorter recording durations}

ICC was used to assess the intra-subject consistency of ARI values for the five distinct recording durations. For the 142 subjects with complete values of ARI for the five durations, ICC was 0.69 (95\% confidence intervals [CI] 0.62-0.75).

Dynamic CA was impaired (ARI $\leq 4$ ) in 14 AIS patients over a 5 min recording duration. An ARI $>4$ with shorter recording durations would represent a false-negative result; rates of 7.1, 7.1, 35.7 and $42.9 \%$ being observed for recording durations of $4,3,2$, and $1 \mathrm{~min}$, respectively (Figure 2a). Similarly, AIS patients with ARI $>4$ over $5 \mathrm{~min}(\mathrm{n}=65)$ could be regarded as 'false-positives' if ARI values were $\leq 4$ on shorter recordings. Again, the number of false-positive results increased from 3.1, 10.8, 12.3 and $15.4 \%$ with a change in recording duration of 4, 3, 2 and $1 \mathrm{~min}$, respectively (Figure. 2b). Though no controls had an ARI $\leq 4$ for recording durations $\geq 4 \mathrm{~min}$, this increased to $2.7 \%, 6.9 \%$ and $16.2 \%$ for durations of 3, 2 and 1 min, respectively (Figure. 2c).

\subsection{Analysis of agreement}

A Bland-Altman plot was used for assessing measurements between 5 min (reference duration) and shorter recording durations (Figure. 3). Table 3 presents the bias and limits of agreement ( \pm 1 .96.SD) for each recording duration, showing significant differences in bias for controls, but not patients. Differences in ARI between 5 and 1 min showed a significant negative trend $\left(\mathrm{R}^{2}=0.53\right)$ with average ARI in controls (Figure. 3a), but not in AIS (Figure. 3b).

\subsection{Parameter sensitivity with alternative window durations in Welch's method}

Changing the window number of samples in Welch's method to 256 and 128 samples for recording durations of 3 and $2 \mathrm{~min}$, respectively, did not significantly change the overall results. However, the number of control subjects with rejected ARI values was reduced (10 vs. 4).

\section{Discussion}

\subsection{Main findings}

Though the influence of recording duration on $\mathrm{dCA}$ estimates has been previously reported (Deegan et al., 2011; Mahdi et al., 2017; Chi et al., 2018), to our knowledge, this is the first study to show the effects of shortening recording duration on ARI estimates in both healthy controls and a disease population. Overall, our results show that the ARI did not change within each group when recording durations were gradually reduced from 5 to $1 \mathrm{~min}$, and that the ICC remained high when taking into account other studies of the reproducibility of dCA metrics (Brodie et al., 2009; Sanders et al., 2018; 
Tzeng et al., 2012). Nevertheless, a number of other factors need to be considered when reducing recording duration in clinical studies, particularly for between-population comparisons.

\subsection{Methodological aspects}

In healthy physiological studies, continuous recordings of $\mathrm{CBFV}, \mathrm{BP}$ and $\mathrm{EtCO}_{2}$ for 5 min or more (Tang et al., 2008; Mahdi et al., 2017; Chi et al., 2017) are relatively straightforward. At the stage of data editing though, large artefacts or signal loss can eventually be identified, resulting in less than the minimum 5 min of signal duration, as recommended by CARNet's White Paper (Claassen et al., 2016) for TFA based studies of dCA. In clinical studies, signal loss and artefacts, compounded by patient discomfort and movement, are a much greater problem, with recordings that maintain good signal quality for $\geq 5$ min being more challenging to obtain. The literature highlights a number of different approaches to simulate the effects of recording duration and signal loss. Deegan et al. (2011) compared the effect of reducing time series duration from 5 to $1 \mathrm{~min}$ on TFA estimates in the $0.03-0.5 \mathrm{~Hz}$ frequency range. Meel-van den Abeelen et al. (2016) illustrated the influence of randomly placed artefacts (loss of signal, motion artefacts and baseline drifts) in CBFV and BP signals on TFA estimates of gain, phase and coherence in selected frequency bands. However, whilst these studies provided alternatives to reducing recording durations and effect of artefacts on TFA outcomes in healthy subjects, effects on patient data, and on the sensitivity of detecting pathological deterioration of dCA, were not established. Mahdi et al. (2017) shortened recordings from $16 \mathrm{~min}$ to $1 \mathrm{~min}$, studying the stability of three parameters (ARI, Mx and TFA phase), and concluded that the minimum recording duration in healthy subjects should not be $<3$ min. Chi et al. (2018) compared dCA indices (Mx and TFA) of AIS patients and controls between 5 and 10 minute signal lengths, but did not report any significant differences between $\mathrm{dCA}$ indices derived with these two different durations. In our study, we used the first 1, 2, 3, or 4 min of a good quality $5 \mathrm{~min}$ recording to simulate the effects of signal loss. This design has the advantage of minimising the influence of nonstationarity of dCA (Panerai, 2014), which could result from comparing shorter segments from different sections of the original 5 min recording. Inevitably, with shorter durations, it was not possible to maintain the recommended duration of $102.4 \mathrm{~s}\left(\mathrm{~N}_{\mathrm{w}}=512\right.$ samples in our case) for segmented data with Welch's method (Claassen et al., 2016). Our choice of combining the number of windows (hence influencing number of degrees of freedom) with reductions in $\mathrm{N}_{\mathrm{w}}$ (hence affecting frequency resolution) aimed to make use of as many segments of $\mathrm{N}_{\mathrm{w}}=512$ as possible, but that could not be maintained satisfactorily for $3 \mathrm{~min}$ or less and for this reason alternatives were considered, without showing a dominant influence on results. Although reducing $\mathrm{N}_{\mathrm{w}}$ can influence estimates of average gain and phase on specific frequency bands (Zhang et al., 1998), it is important to note that this does not apply to ARI, which tends to remain relatively constant when changing $\mathrm{N}_{\mathrm{w}}$ (Panerai et al., 2016a). 
On theoretical grounds, reducing the length of data in TFA increases the bias and variance of estimated parameters, like the gain and phase, independently of the physical or biological origin of the data (Bendat and Piersol, 2011). For signals reflecting cerebrovascular control though, this effect can be exacerbated by the contribution of non-stationarity or co-variates, such as arterial $\mathrm{CO}_{2}$, sensorimotor, or cognitive neural stimulation (Panerai, 2014). Although the partial, theoretical effect of reducing data length could be estimated for different parameters, assuming the data were generated by a stationary process (Bendat and Piersol, 2011), the integrated approach we adopted, as reflected by Figure 1, is preferable as it provides the overall effects of reducing recording duration, that includes any potential contributions of nonstationarity or other physiological co-variates.

In ideal conditions, the dependence of sensitivity and specificity of different dCA metrics on recording duration would be of considerable interest to establish minimum acceptable durations in clinical studies. Unfortunately, in the absence of a standard reference for dCA, it was not possible to construct ROC curves in our study, as not all AIS patients can be assumed to have impaired dCA. This is different from other studies, such as Katsogridakis et al. (2013), where hypercapnia was used as a surrogate of impaired dCA, and hence assumed to affect all subjects in the group. Nevertheless, using an empirical criterion (ARI $\leq 4$ ), we have shown that reducing recording duration to $3 \mathrm{~min}$ had a relatively small effect on false-positive and false-negative rates, whilst this effect was considerably more deleterious for durations of $\leq 2 \mathrm{~min}$. Future studies will need to re-assess these findings, if different criteria are used to classify patients with impaired dCA.

\subsection{Clinical implications}

The influence of reducing recording duration in AIS patients, below the 5 min recommended by the White paper (Claassen et al., 2016), has not been reported previously. Although Mahdi et al (2017) studied only healthy subjects, and used a different metric to assess the influence of shorter recordings, their conclusions were similar to ours: recordings with less than 3 min duration should not be used in applications of TFA for dCA assessment.

Our conclusions followed from the permanence of significant differences in ARI values between AIS and controls (Figure. 1, Table 2), as well as the sharp rise in false-positive and false-negative rates for recordings shorter than 3 min (Figure. 2).

The conclusion that recordings with 3 min duration might be acceptable for clinical studies of dCA, does not imply that this duration should be standard in data collection protocols. On the contrary, whenever technically possible, and subject to patients' comfort and consent, measurements longer than 5 min should be targeted to allow for the possibility of selecting segments with optimal data quality for TFA. We emphasize that reduced duration, down to a minimum of 3 min should always be regarded as 
the exception, not the rule. Moreover, it is important to keep in mind that recordings with 3 min duration incur a false-positive rate of $10.8 \%$ in comparison with results obtained with the 5 min standard.

\subsection{Limitations of the study}

There are several limitations in our study. CBFV can only reflect changes in absolute CBF as long as the diameter of MCA remains constant. This is likely to be the case with subjects at rest and in normocapnia (Table 1). Even though all data were recorded with a similar protocol, and in the same research laboratory, this is a retrospective observational study with data collected by different TCD operators and this might have contributed to a wider distribution of values of CBFV and other parameters. However, the main parameter in this study, namely ARI, is independent of the absolute amplitudes of BP and CBFV, being only dependent on the temporal pattern of the CBFV step response. Although this study did not explicitly present TFA results for coherence, phase and gain, coherence was implicitly incorporated in the acceptance criteria for ARI (Panerai et al., 2016a). Moreover, all the frequency components of gain and phase were used with the inverse FFT to obtain the CBFV step response, from where ARI values were derived.

The reliability of TFA estimates of CA parameters is likely to depend on BP variability and this will tend to be reduced with shorter recording durations (Claassen et al., 2016; Katsogridakis et al., 2013). However, we have not analysed the role of this co-factor in our study, to be consistent with the "nochoice' aspect of simulating the use of whatever data are available, instead of the alternative of being able to choose segments with the largest BP variability. By using the reduced duration segments always at the beginning of the longer $5 \mathrm{~min}$ recording, one would expect that the influence of BP variability would be randomly distributed across subjects and patients, and hence not have affected the main results significantly. Nevertheless, this is a relevant aspect of TFA analysis that deserves further investigation.

Five patients had posterior circulation strokes (POCS), and were not expected to show alterations in dCA based on MCA measurements, since the posterior circulation is supplied by the vertebrobasilar system. Nevertheless, it has been shown that lesions of the posterior circulation can have a diffuse effect on both hemispheres, and for this reason patients with POCS can still show alterations when assessed in the MCA (Guo et al., 2015). Moreover, when analysed separately, these patients did not show any distinct behaviour from those with partial anterior strokes.

Finally, it is important to emphasize that the results of this study are limited to the use of TFA within the set of parameters described above and cannot be extended to other approaches for modelling the BP-CBFV dynamic relationship such as time-domain techniques (Czosnyka et al., 2008; Mahdi et al., 2017; Nogueira et al., 2013; Panerai et al., 2001; Panerai et al., 2016b; Sanders et al., 2018; Tzeng et al., 2012) or closed-loop models as proposed by Marmarelis et al. (2013). 


\section{Conclusions}

The possibility of using reduced recording durations, down to a minimum of $3 \mathrm{~min}$, when good quality measurements of the recommended minimum 5 min duration are not possible, may substantially impact on the translation of methods of $\mathrm{dCA}$ assessment into clinical practice and, in turn, improve the individualised care of stroke patients. Further work is needed to investigate the applicability of these findings to other disease populations.

\section{Acknowledgements}

Supported by EPSRC grant EP/K041207/1. The authors would like to thank the Ministry of Sciences and Technology of the Royal Thai Government and Thammasat University (Thailand) for supporting a $\mathrm{PhD}$ scholarship for the first author. 
Table 1. Demographic and baseline physiological parameters.

\begin{tabular}{|c|c|c|c|}
\hline & Healthy controls & Stroke patients & P-value \\
\hline Number (n) & 78 & 79 & \\
\hline Mean Age (SD), years & $52.0(16.3)$ & $67.5(12.2)$ & $<0.0001$ \\
\hline Gender: male (\%) & $41(52.6 \%)$ & $55(69.6 \%)$ & 0.0338 \\
\hline $\operatorname{CBFV}(\mathrm{cm} / \mathrm{s}), R$ and $A H$ & $52.7(13.8)$ & $44.9(15.7)$ & 0.0002 \\
\hline $\operatorname{CBFV}(\mathrm{cm} / \mathrm{s}), L$ and $U H$ & $52.2(17.1)$ & $42.3(18.9)$ & $<0.0001$ \\
\hline$M A P(m m H g)$ & $87.8(14.2)$ & $100.7(15.0)$ & $<0.0001$ \\
\hline$H R(b p m)$ & $64.0(10.3)$ & $71.3(12.1)$ & $<0.0001$ \\
\hline $\mathrm{EtCO}_{2}(\mathrm{mmHg})$ & $38.8(4.0)$ & $27.8(12.8)$ & $<0.0001$ \\
\hline Affected hemisphere (R/L) & - & $33 / 46$ & - \\
\hline Mean NIHSS score (SD) & - & $4.4(3.8)$ & - \\
\hline \multicolumn{4}{|l|}{ Stroke subtype (\%) } \\
\hline TACS & - & $8(10.1 \%)$ & - \\
\hline POCS & - & $5(6.3 \%)$ & - \\
\hline PACS & - & $37(46.8 \%)$ & - \\
\hline LACS & - & $29(36.7 \%)$ & - \\
\hline
\end{tabular}

$n$, number of participants; $R$, right; $L$, left; $A H$, affected hemisphere; $U H$, unaffected hemisphere; NIHSS, National Institute of Health Stroke Scale (on the day of dCA assessment); CBFV, cerebral blood flow velocity; MAP, mean arterial blood pressure; $\mathrm{HR}$, heart rate; $\mathrm{EtCO}_{2}$, end-tidal carbon dioxide; TACS, Total Anterior Circulation Stroke; POCS, Posterior Circulation Stroke; PACS, Partial Anterior Circulation Stroke; LACS, Lacunar Stroke; bpm, beats per minute.

Table 2. The mean values (SD) of TFA parameters and comparison of dCA assessment for each recording duration.

\begin{tabular}{|c|c|c|c|c|c|}
\hline ARI & $5 \mathrm{~min}$ & $4 \mathrm{~min}$ & $3 \mathrm{~min}$ & $2 \mathrm{~min}$ & $1 \mathrm{~min}$ \\
\hline Healthy controls & $6.7(0.8)(n=78)$ & $6.6(0.9)(n=77)$ & $6.4(1.1)(n=73)$ & $6.3(1.5)(n=73)$ & $6.0(1.9)(n=74)$ \\
\hline AIS patients & $5.6(1.8) *(n=79)$ & $5.6(1.9) *(n=79)$ & $5.6(1.9) *(n=79)$ & $5.6(2.1)(n=78)$ & $5.4(2.2)(n=76)$ \\
\hline
\end{tabular}

ARI, cerebral autoregulation index. ( $n$ ): number of accepted values of ARI for each recording duration.

* $p<0.05$ compared to controls.

Table 3. Bland-Altman analysis of agreement for autoregulation index for shorter recording durations in comparison with a 5-min recording reference.

\begin{tabular}{|c|c|c|c|c|}
\hline$A R I$ & $5 \mathrm{~min}$ vs $4 \mathrm{~min}$ & $5 \mathrm{~min}$ vs $3 \mathrm{~min}$ & $5 \mathrm{~min}$ vs $2 \mathrm{~min}$ & $5 \mathrm{~min}$ vs $1 \mathrm{~min}$ \\
\hline Healthy controls & $\begin{array}{c}0.112(0.4[-0.6-0.8])^{*} \\
(n=77)\end{array}$ & $\begin{array}{c}0.235(0.6[-1.0-1.5])^{*} \\
(n=73)\end{array}$ & $\begin{array}{c}0.327(1.3[-2.1-2.8])^{*} \\
(n=73)\end{array}$ & $\begin{array}{c}0.768(1.8[-2.8-4.3])^{*} \\
(n=74)\end{array}$ \\
\hline AlS patients & $\begin{array}{c}-0.065(0.7[-1.4-1.2]) \\
(n=79)\end{array}$ & $\begin{array}{c}-0.009(1.3[-2.5-2.5]) \\
(n=79)\end{array}$ & $\begin{array}{c}-0.105(1.6[-3.2-3.0]) \\
(n=78)\end{array}$ & $\begin{array}{c}0.163(1.7[-3.2-3.6]) \\
(n=76)\end{array}$ \\
\hline
\end{tabular}

Values are the bias (SD and [Limits of Agreement]). ARI, cerebral autoregulation index. (n): number of accepted values of ARI for each duration. ${ }^{*} p<0.05$ for differences in bias compared to the first 5 min. 


\section{Figure legends}

Figure 1. Population mean ARI and SEM (error bars) of the autoregulation index (ARI) for different recording durations. Healthy controls (dark grey) and stroke patients (light grey). ${ }^{*} p<0.05$ for difference between strokes and healthy controls.

Figure 2. Percentage of AIS patients classified as (a) false-negatives when ARI $>4$ for reduced recording durations, based on the original classification of impaired dynamic CA (ARI $\leq 4)$ in the 5 min recording and (b) false-positives with ARI $\leq 4$ that had ARI $>4$ in 5 min recording. (c) Percentage of healthy controls with ARI $\leq 4$ in shorter recording durations. AIS: acute ischaemic stroke, HC: healthy control.

Figure 3. Bland-Altman plots of ARI estimates with linear regression for (a) control subjects and (b) AIS patients, comparing durations of $1 \mathrm{~min}$ with the $5 \mathrm{~min}$ reference. The lower and upper limits of agreements are the dash lines and the bias (mean difference) is the dotted line. 
Figure 1.

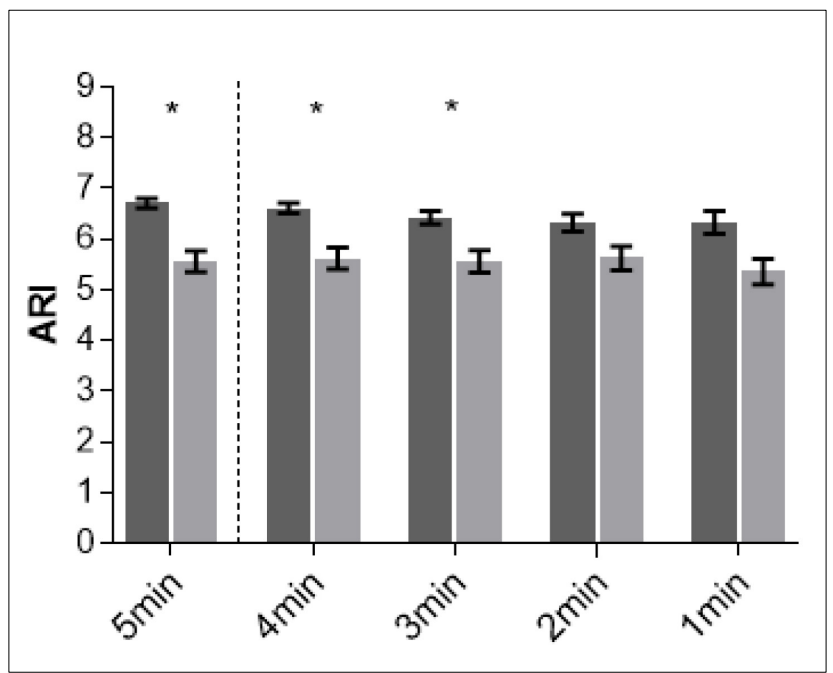

Figure 2.
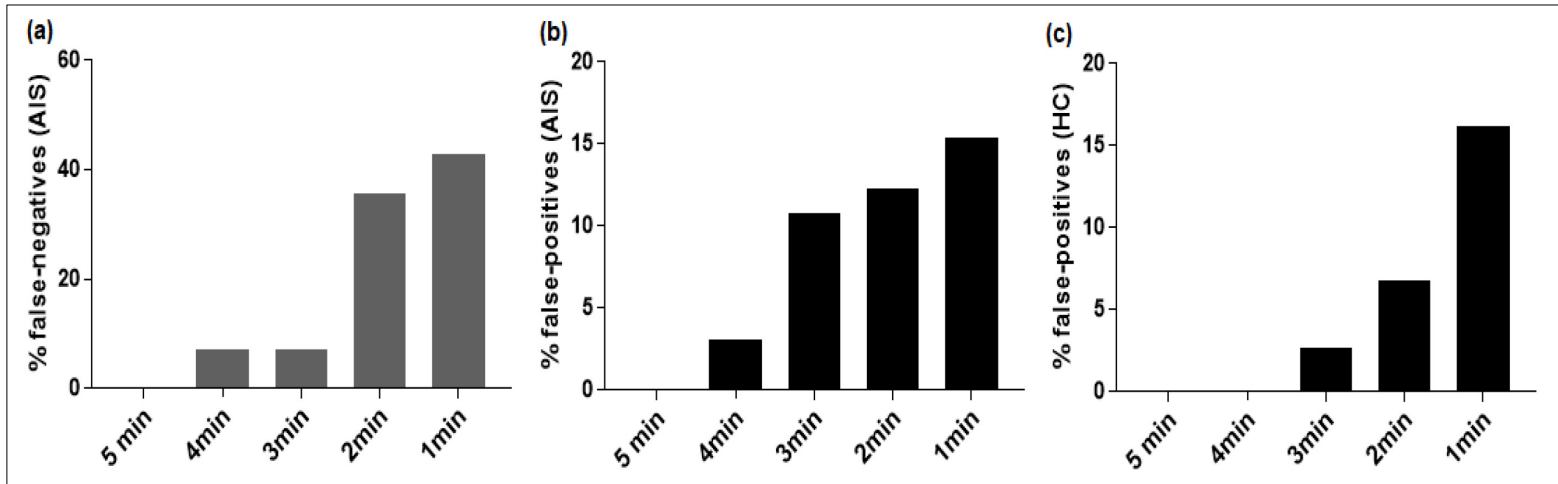

Figure 3.
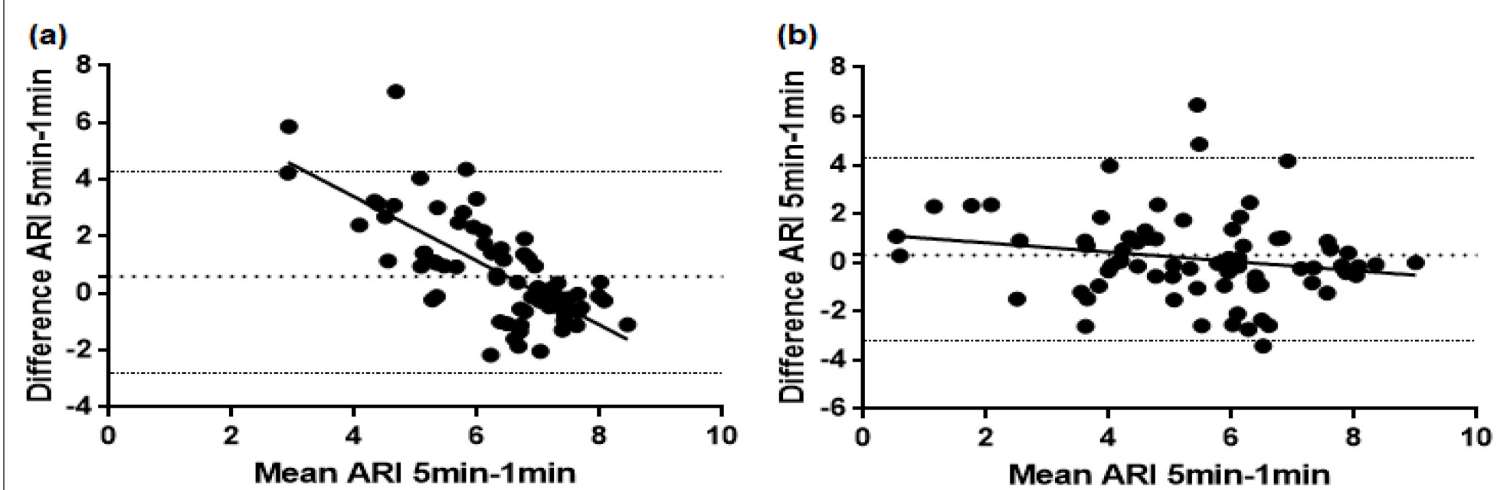


\section{References}

Aaslid R, Lindegaard K F, Sorteberg W and Nornes H 1989 Cerebral autoregulation dynamics in humans Stroke 20 45-52

Aries M J, Elting J W, De Keyser J, Kremer B P and Vroomen P C 2010 Cerebral autoregulation in stroke: a review of transcranial Doppler studies Stroke 41 2697-704

Atkins E R, Brodie F G, Rafelt S E, Panerai R B and Robinson T G 2010 Dynamic cerebral autoregulation is compromised acutely following mild ischaemic stroke but not transient ischaemic attack Cerebrovasc Dis 29 228-35

Bendat J S and Piersol A G 2011 Random data: analysis and measurement procedures $4^{\text {th }}$ edn vol 729 : John Wiley \& Sons

Bland J M and Altman D G 1986 Statistical Methods for Assessing Agreement between Two Methods of Clinical Measurement Lancet 1 307-10

Brodie F G, Atkins E R, Robinson T G and Panerai R B 2009 Reliability of dynamic cerebral autoregulation measurement using spontaneous fluctuations in blood pressure Clin Sci (Lond) $116513-20$

Caldas J R, Panerai R B, Bor-Seng-Shu E, Ferreira G S R, Camara L, Passos R H, de-Lima-Oliveira M, Galas F, Almeida J P, Nogueira R C, Mian N, Gaiotto F A, Robinson T G and Hajjar L A 2019 Dynamic cerebral autoregulation: A marker of post-operative delirium? Clin Neurophysiol 130 101-8

Caldas J R, Panerai R B, Haunton V J, Almeida J P, Ferreira G S, Camara L, Nogueira R C, Bor-Seng-Shu E, Oliveira M L, Groehs R R, Ferreira-Santos L, Teixeira M J, Galas F R, Robinson T G, Jatene F B and Hajjar L A 2017 Cerebral blood flow autoregulation in ischemic heart failure Am J Physiol Regul Integr Comp Physiol 312 R108-R13

Carey B J, Manktelow B N, Panerai R B and Potter J F 2001 Cerebral autoregulatory responses to headup tilt in normal subjects and patients with recurrent vasovagal syncope Circulation 104 898902

Carey B J, Panerai R B and Potter J F 2003 Effect of aging on dynamic cerebral autoregulation during head-up tilt Stroke 34 1871-5

Chi N F, Ku H L, Wang C Y, Liu Y, Chan L, Lin Y C, Peng C K, Novak V, Hu H H and Hu C J 2017 Dynamic Cerebral Autoregulation Assessment Using Extracranial Internal Carotid Artery Doppler Ultrasonography Ultrasound Med Biol 43 1307-13

Chi N F, Wang C Y, Chan L and Hu H H 2018 Comparing Different Recording Lengths of Dynamic Cerebral Autoregulation: 5 versus 10 Minutes Biomed Res Int 20187803426

Claassen J A, Meel-van den Abeelen A S, Simpson D M, Panerai R B and international Cerebral Autoregulation Research N 2016 Transfer function analysis of dynamic cerebral autoregulation: A white paper from the International Cerebral Autoregulation Research Network J Cereb Blood Flow Metab 36 665-80

Czosnyka M, Smielewski P, Lavinio A, Pickard J D and Panerai R 2008 An assessment of dynamic autoregulation from spontaneous fluctuations of cerebral blood flow velocity: a comparison of two models, index of autoregulation and mean flow index Anesth Analg 106 234-9, table of contents

Deegan B M, Devine E R, Geraghty M C, Jones E, Olaighin G and Serrador J M 2010 The relationship between cardiac output and dynamic cerebral autoregulation in humans J Appl Physiol (1985) 109 1424-31

Deegan B M, Serrador J M, Nakagawa K, Jones E, Sorond F A and Olaighin G 2011 The effect of blood pressure calibrations and transcranial Doppler signal loss on transfer function estimates of cerebral autoregulation Med Eng Phys 33 553-62

Giller C A 1990 The frequency-dependent behavior of cerebral autoregulation Neurosurgery 27 362-8

Guo Z N, Xing Y, Wang S, Ma H, Liu J and Yang Y 2015 Characteristics of dynamic cerebral autoregulation in cerebral small vessel disease: Diffuse and sustained Sci Rep $\mathbf{5} 15269$

Haunton V J 2014 Is dynamic cerebral autoregulation impaired in idiopathic Parkinson's disease? : MD Thesis, Department of Cardiovascular Sciences, University of Leicester 
Katsogridakis E, Bush G, Fan L, Birch A A, Simpson D M, Allen R, Potter J F and Panerai R B 2013 Detection of impaired cerebral autoregulation improves by increasing arterial blood pressure variability J Cereb Blood Flow Metab 33 519-23

Katsogridakis E, Dineen N E, Brodie F G, Robinson T G and Panerai R B 2011 Signal-to-noise ratio of bilateral nonimaging transcranial Doppler recordings of the middle cerebral artery is not affected by age and sex Ultrasound Med Biol 37 530-8

Lam M Y, Haunton V J, Robinson T G and Panerai R B 2019 Dynamic cerebral autoregulation measurement using rapid changes in head positioning: experiences in acute ischemic stroke and healthy control populations Am J Physiol Heart Circ Physiol 316 H673-H83

Lassen N A 1959 Cerebral blood flow and oxygen consumption in man Physiol Rev 39 183-238

Llwyd O, Panerai R B and Robinson T G 2017 Effects of dominant and non-dominant passive arm manoeuvres on the neurovascular coupling response Eur J Appl Physiol 117 2191-9

Llwyd O, Salinet A S M, Panerai R B, Lam M Y, Saeed N P, Brodie F, Bor-Seng-Shu E, Robinson T G and Nogueira R C 2018 Cerebral Haemodynamics following Acute Ischaemic Stroke: Effects of Stroke Severity and Stroke Subtype Cerebrovasc Dis Extra 8 80-9

Mahdi A, Nikolic D, Birch A A and Payne S J 2017 At what data length do cerebral autoregulation measures stabilise? Physiol Meas 38 1396-404

Marmarelis V Z, Shin D C, Orme M E and Zhang R 2013 Closed-loop dynamic modeling of cerebral hemodynamics Ann Biomed Eng 41 1029-48

Meel-van den Abeelen A S, de Jong D L, Lagro J, Panerai R B and Claassen J A 2016 How measurement artifacts affect cerebral autoregulation outcomes: A technical note on transfer function analysis Med Eng Phys 38 490-7

Nakagawa K, Serrador J M, Larose S L, Moslehi F, Lipsitz L A and Sorond F A 2009 Autoregulation in the posterior circulation is altered by the metabolic state of the visual cortex Stroke 40 2062-7

Nogueira R C, Bor-Seng-Shu E, Santos M R, Negrao C E, Teixeira M J and Panerai R B 2013 Dynamic cerebral autoregulation changes during sub-maximal handgrip maneuver Plos One 8 e70821

Panerai R B 1998 Assessment of cerebral pressure autoregulation in humans--a review of measurement methods Physiol Meas 19 305-38

Panerai R B 2008 Cerebral autoregulation: from models to clinical applications Cardiovasc Eng 8 42-59

Panerai R B 2014 Nonstationarity of dynamic cerebral autoregulation Med Eng Phys 36 576-84

Panerai R B, Dawson S L, Eames P J and Potter J F 2001 Cerebral blood flow velocity response to induced and spontaneous sudden changes in arterial blood pressure Am J Physiol Heart Circ Physiol 280 H2162-74

Panerai R B, Haunton V J, Hanby M F, Salinet A S and Robinson T G 2016a Statistical criteria for estimation of the cerebral autoregulation index (ARI) at rest Physiol Meas 37 661-72

Panerai R B, Hudson V, Fan L, Mahony P, Yeoman P M, Hope T and Evans D H 2002 Assessment of dynamic cerebral autoregulation based on spontaneous fluctuations in arterial blood pressure and intracranial pressure Physiol Meas 23 59-72

Panerai R B, Jara J L, Saeed N P, Horsfield M A and Robinson T G 2016b Dynamic cerebral autoregulation following acute ischaemic stroke: Comparison of transcranial Doppler and magnetic resonance imaging techniques J Cereb Blood Flow Metab 36 2194-202

Panerai R B, White R P, Markus H S and Evans D H 1998 Grading of cerebral dynamic autoregulation from spontaneous fluctuations in arterial blood pressure Stroke 29 2341-6

Patel N, Panerai R B, Haunton V, Katsogridakis E, Saeed N P, Salinet A, Brodie F, Syed N, D'Sa S and Robinson T G 2016 The Leicester cerebral haemodynamics database: normative values and the influence of age and sex Physiol Meas 37 1485-98

Puppo C, Lopez L, Caragna E and Biestro A 2008 One-minute dynamic cerebral autoregulation in severe head injury patients and its comparison with static autoregulation. A transcranial Doppler study Neurocrit Care 8 344-52 
Saeed N P, Panerai R B, Horsfield M A and Robinson T G 2013 Does stroke subtype and measurement technique influence estimation of cerebral autoregulation in acute ischaemic stroke? Cerebrovasc Dis 35 257-61

Salinet A S, Panerai R B and Robinson T G 2014 The longitudinal evolution of cerebral blood flow regulation after acute ischaemic stroke Cerebrovasc Dis Extra 4 186-97

Sanders M L, Claassen J, Aries M, Bor-Seng-Shu E, Caicedo A, Chacon M, Gommer E D, Van Huffel S, Jara J L, Kostoglou K, Mahdi A, Marmarelis V Z, Mitsis G D, Muller M, Nikolic D, Nogueira R C, Payne S J, Puppo C, Shin D C, Simpson D M, Tarumi T, Yelicich B, Zhang R, Panerai R B and Elting J W J 2018 Reproducibility of dynamic cerebral autoregulation parameters: a multicentre, multi-method study Physiol Meas 39125002

Shrout P E and Fleiss J L 1979 Intraclass correlations: uses in assessing rater reliability Psychol Bull 86 420-8

Subudhi A W, Grajzel K, Langolf R J, Roach R C, Panerai R B and Davis J E 2015 Cerebral autoregulation index at high altitude assessed by thigh-cuff and transfer function analysis techniques Exp Physiol 100 173-81

Tang S C, Huang Y W, Shieh J S, Huang S J, Yip P K and Jeng J S 2008 Dynamic cerebral autoregulation in carotid stenosis before and after carotid stenting J Vasc Surg 48 88-92

Tiecks F P, Lam A M, Aaslid R and Newell D W 1995 Comparison of static and dynamic cerebral autoregulation measurements Stroke 26 1014-9

Tzeng Y C, Ainslie P N, Cooke W H, Peebles K C, Willie C K, MacRae B A, Smirl J D, Horsman H M and Rickards C A 2012 Assessment of cerebral autoregulation: the quandary of quantification $\mathrm{Am}$ J Physiol Heart Circ Physiol 303 H658-71

Wahlgren N, Ahmed N, Davalos A, Ford G A, Grond M, Hacke W, Hennerici M G, Kaste M, Kuelkens S, Larrue V, Lees K R, Roine R O, Soinne L, Toni D, Vanhooren G and investigators S-M 2007 Thrombolysis with alteplase for acute ischaemic stroke in the Safe Implementation of Thrombolysis in Stroke-Monitoring Study (SITS-MOST): an observational study Lancet $\mathbf{3 6 9}$ 275-82

Weir J P 2005 Quantifying test-retest reliability using the intraclass correlation coefficient and the SEM J Strength Cond Res 19 231-40

Zhang R, Zuckerman J H, Giller C A and Levine B D 1998 Transfer function analysis of dynamic cerebral autoregulation in humans Am J Physiol Heart Circ Physiol 274 H233-41 